Presented at Hadron 97, Brookhaven National Laboratory, Upton, New York

August 25, 1997

BNL- 65030

$$
\text { CONF-970842-- }
$$

\title{
Measurement of Color Transparency by $C(p, 2 p)$ Reactions at Large Momentum Transfer
}

\author{
A. S. Carroll* \\ AGS Department, Brookhaven National Laboratory
}

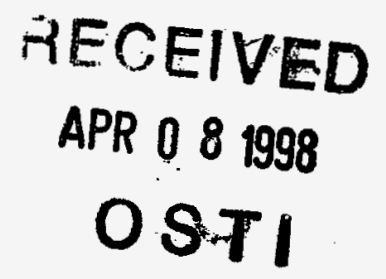

The subject of color transparency, the enhancement of the ability of hadrons to penetrate nuclear matter by kinematic selection, is both interesting and controversial. The description of the collision of hadrons with nucleons inside nuclei, and the connection with initial and final state interactions involve fundamental questions of quantum mechanics, and nuclear and particle physics. Interest in color transparency was greatly increased by AGS Experiment 834 which observed dramatic changes with incident momentum for a variety of nuclei. ${ }^{1}$ A new experiment, E850, has studied the $(p, 2 p)$ quasi-elastic reaction near $90^{\circ} \mathrm{cm}$ for momenta between 5.9 and $9 \mathrm{GeV} / \mathrm{c}$. The quasi-elastic reaction was compared to the elastic reaction on free protons to determine the transparency. In simple terms, we define the transparency ratio as

$$
\operatorname{Tr}=d \sigma / d t(p p, \text { in nucleus }) / d \sigma / d t(p p, \text { free protons }) \ldots . . .
$$

The E834 collaboration with the addition of a number of new experimenters designed and built a solenoidal spectrometer for E850 in order to continue this investigation of color transparency and many other phenomena related to exclusive reactions at large momenta transfer. This new spectrometer, as shown in Fig 1, allowed us to measure the momenta for all particles emitted in a broad angular region about $90^{\circ} \mathrm{cm}$. In contrast, E834 could only measure the momentum of one outgoing particle. This lack of kinematical constraints lead to a significant background in the E834 quasi-elastic sample.

* Representing the E850 collaboration: H. Aclander ${ }^{1}$, J. Alster ${ }^{1}$, D. Barton ${ }^{2}$, G. Bunce ${ }^{2}$, N. Christensen ${ }^{3}$, H. Courant ${ }^{3}$, S. Durrant ${ }^{2}$, S. Gushue ${ }^{2}$, S. Heppel$\operatorname{mann}^{4}$, I. Mardor ${ }^{1}, Y$. Mardor $^{1}$, M. Marshak ${ }^{3}$, Y. Makdisi ${ }^{2}$, E. Minor ${ }^{4}$, H. Nicholson ${ }^{5}$, E. Piasetzky ${ }^{1}$ T. Roser ${ }^{2}$, J. Russell ${ }^{6}$, S. Sutton ${ }^{5}$, M. Tanaka ${ }^{2+}$, C. White ${ }^{3}, \mathrm{~J}-\mathrm{Y} \mathrm{Wu} \mathrm{u}^{4}$.

${ }^{1}$ Tel Aviv University, ${ }^{2}$ Brookhaven National Laboratory, ${ }^{3}$ University of Minnesota, ${ }^{4}$ Pennsylvania State University, ${ }^{5}$ Mount Holyoke College, ${ }^{6}$ University of Massachusetts Dartmouth, ${ }^{+}$deceased

The data analysis was done largely by S. Durrant and I. Mardor. Details to appear in the PhD thesis of I. Mardor.

*This work was performed under the auspices of the U.S. Department of Energy under Contract No. DE-ACO2-76CH00016. 


\section{DISCLAIMER}

This report was prepared as an account of work sponsored by an agency of the United States Government. Neither the United States Government nor any agency thereof, nor any of their employees, makes any warranty, expreas or implied, or assumes any legal liability or responsibility for the accuracy, completeness, or usefulness of any information, apparatus, product, or process disclosed, or represents that its use would not infringe privately owned rights. Reference herein to any specific commercial product, process, or service by trade name, trademark, manufacturer, or otherwise does not necessarily constitute or imply its endorsement, recommendation, or favoring by the United States Government or any agency thereof. The views and opinions of authors expressed herein do not necessarily state or reflect those of the United States Government or any agency thereof. 
Unfortunately, the operation of the super-conducting solenoid has been quite erratic, so that there has been only one season of moderately sustained data taking in 1994. The data presented here represents color transparency measurements using Carbon(C), $C H_{2}$ and $C D_{2}$ targets at 5.9, 7.5 and $9 \mathrm{GeV} / \mathrm{c}$.

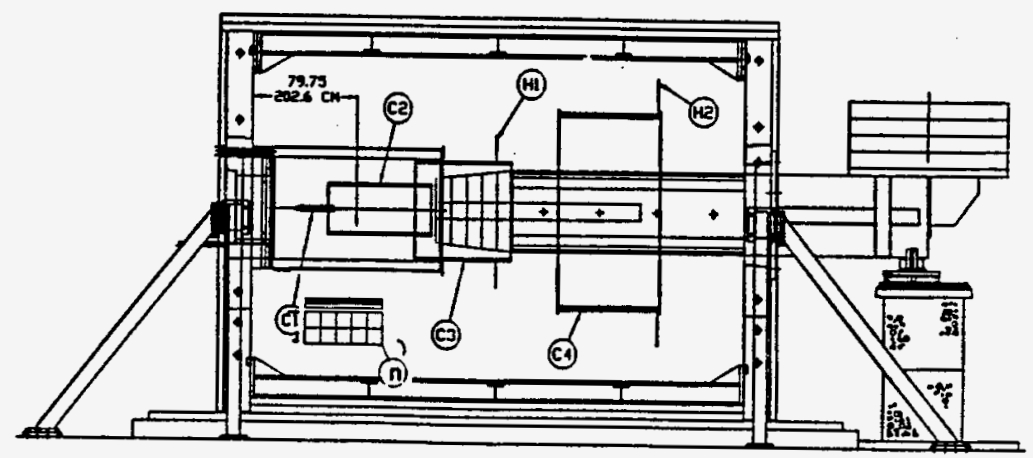

Fig 1. Schematic of EVA Spectrometer for E850

We selected events corresponding to elastic scattering free protons in the $\mathrm{CH}_{2}$ target, or quasi-elastic from protons in deuterium or Carbon. The selection criteria included the absence of other tracks associated with the event; limits on the transverse components of the target proton momentum(in plane component, $p_{x}<0.5 \mathrm{GeV} / \mathrm{c}$ and out of reaction plane component, $p_{y}$ $<0.3 \mathrm{GeV} / \mathrm{c}$ ); limits on the longitudinal component parameterized by the light cone variable, $0.95<\alpha<1.05$; missing energy between the initial and two measured outgoing particles, $E_{\text {miss }}<0.5 \mathrm{GeV}$.

The transparency ratios quoted in this paper are the number of quasi-elastic events within the kinematic cuts list above compared to free proton events in the $\mathrm{CH}_{2}$ targets selected by the same criteria. There is no attempt to try to calculate the entire number of quasi-elastic events integrated over the full range of target(Fermi) momenta as in E834.

The number of events from the $\mathrm{CH}_{2}$ target is the number of hydrogen events plus the number from $\mathrm{C}$ within the linematic cuts. If we form the ratio of $\mathrm{C}$ to $\mathrm{CH}_{2}$ events $\mathrm{R}(\mathrm{C} / \mathrm{H})$, then the Transparency ratio for $\mathrm{C}$ events divided by hydrogen events, $\operatorname{Tr}(\mathrm{C} / \mathrm{H})$, can be calculated as

$$
\operatorname{Tr}(\mathrm{C} / \mathrm{H})=\mathrm{R}(\mathrm{C} / \mathrm{H}) /(\beta-\mathrm{R}(\mathrm{C} / \mathrm{H})) \ldots . .(2)
$$

where $\beta$ is the ratio of the $\mathrm{C}$ scatterers in the two targets, 2.189 .

In order to minimize systematic errors due to acceptance and beam normalization, we analyzed the targets in pairs as shown in the table below. The distance between the centers of the three, $6 \mathrm{~cm}$ long targets was about 22 cm. All three targets were in the beam simultaneously, and received the same beam flux. Their order was changed periodically.

By pairing the data sets from Mid and Downstream targets from Data Set 1 with the corresponding pair from Data Set 2, we can obtain an expression 
for the $\mathrm{C}$ to $\mathrm{CH}_{2}$ ratio which is independent of the beam flux, and cancels out the dependence on acceptance for the two targets. Similarly, ratios can be formed combining the results from each target and then cancelling the beam flux.

$\begin{array}{cccc}\text { Data Set } & & & \\ 1 & C D_{2} & C & C H_{2} \\ 2 & C D_{2} & C H_{2} & C \\ 3 & C & C H_{2} & C D_{2} \\ 4 & C & C D_{2} & C H_{2}\end{array}$

Data sets 3 and 4 can be used in a similar way to derived expressions for the deuterium(D) to hydrogen(H) ratio. The kinematic selection cuts listed above include essentially all of the Fermi distribution for the deuteron. The $\mathrm{D} / \mathrm{H}$ transparencies measured are unity within experimental errors. Also these transparencies show no energy dependence as expected for the essentially transparent deuterium nucleus.

In the upper portion of Fig 2, we plot our three preliminary values of the $\mathrm{C}$ transparency ratio. This ratio includes only the portion of the $\mathrm{C}$ spectral function near the hydrogen elastic peak defined by the selection cuts.

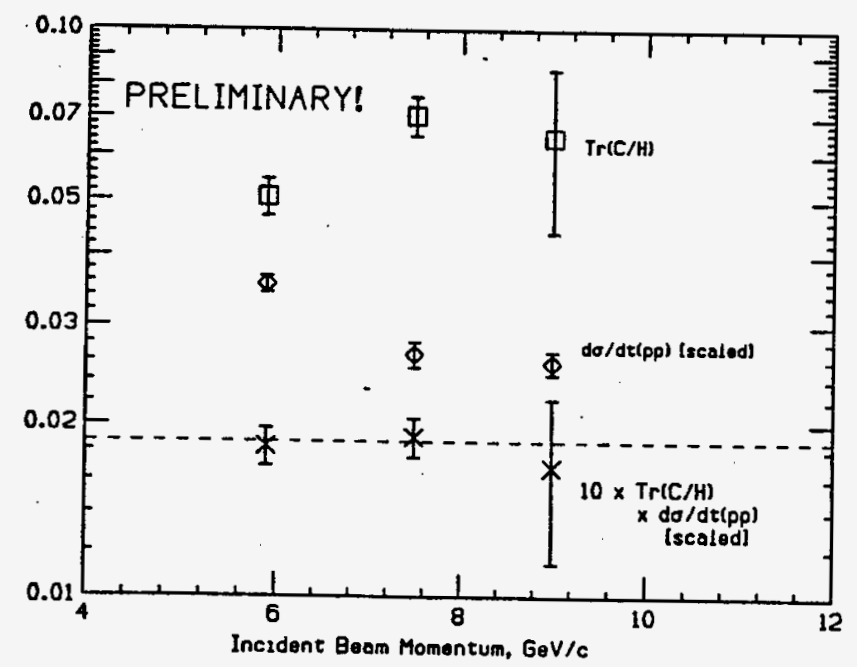

Fig 2. Transparency Ratios, Scaled Cross Sections and Product

We note that there is a rapid rise in the transparency ration between 5.9 and $7.5 \mathrm{GeV} / \mathrm{c}$. Unfortunately, no conclusion can be formed on the energy dependence beyond $7.5 \mathrm{GeV} / \mathrm{c}$, due to the large statistical error on the 9 $\mathrm{GeV} / \mathrm{c}$ data point. 
As noted in equation 1, the transparency satio is the ratio of the quasi-elastic to elastic cross sections. One can ask whether the numerator or the denominator is responsible for the energy variation. The free $90^{\circ} \mathrm{pp}$ differential cross section measured by Akerlof, et $\mathrm{al}^{2}$ has been scaled by $[\mathrm{s} / \mathrm{s}(10 \mathrm{GeV} / \mathrm{c})]^{10}$ and plotted in the lower portion of Fig 2. We see an $s$ dependent behavior in the scaled cross section which is the inverse of the behavior of the transparency ratio. If we form the product of the free pp cross section with the transparency ratio, the result is a quantity which is proportional to the pp cross section in the $\mathrm{C}$ nucleus. We see that this quantity plotted in the center of Fig 2 is consistent with no energy dependence at all.

A theoretical picture emphasized by Ralston and $\mathrm{Pire}^{3}$, is that there are two components to the pp scattering amplitude at large momentum transfer. There is a small perturbative amplitude which results in a cross section which scales as $\mathrm{s}^{-10}$. In addition there is large amplitude which does not follow the scaling behavior, but is "filtered" away in the nuclear matter of the $C$ nucleus. Thus the quasi-elastic cross section in Carbon follows the scaling law within errors.

Further measurements with better statistics and at higher momentum with the EVA detector should help clarify this picture or suggest alternative interpretations ${ }^{4}$.

\section{SUMMARY}

1. With limited statistics, but with better kinematic definition in a new detector, we have confirmed the rise in Carbon transparency ratio seen in Expt 834. The $\operatorname{Tr}(\mathrm{D} / \mathrm{H})$ for deuterium is consistent with no energy dependence.

2. Unlike the free $\mathrm{d} \sigma / \mathrm{dt}$ for hydrogen, the $\mathrm{d} \sigma / \mathrm{dt}$ from protons in a nucleus is consistent with the exact $s^{-10}$ scaling. This suggests two components to the pp scattering amplitude; one small and perturbative, the other spatially large and varying, but "filtered" away by the nuclear matter in the Carbon nucleus.

3. Our plan is to complete the repairs of the super-conducting solenoid early this fall ${ }^{++}$, reassemble the detector, and collect data starting next spring.

++ The EVA super-conducting solenoid successfully reached operating current again on 6 November $199 \%$.

\section{REFERENCES}

1. A. S. Carroll et al, Phys. Rev Lett. 61,1698(1988)

2. C. W. Akerlof et al,Phys. Rev. 159,1138(1967)

3. J.P. Ralston and B. Pire, Phys. Rev Lett. 61,1823(1988)

4. L. Frankfurt, G.A. Miller, and M. Strikman, Comment Nucl. Part. Physics 21,1(1992) 
M98004428

|||||||||||||||||||||||||||||||||||||||||||||||||||||

Report Number (14) BNL-- 65030

CONF $-970842 \ldots$

Publ. Date (11) $1997 / 2$

Sponsor Code (18) DOE/ER

UC Category (19) $U C-414, D O E / E R$ 